\title{
Understanding of the Earth from Annular Eclipses of the Sun
}

The annular eclipse of January 15, 2010 created lot of interest among many here in India. All those who travelled to the southern tip enjoyed the beautiful sight of the Sun turning into a glowing bracelet. The accompanying chill weather and the circles beneath the trees added to the unique experience. No wonder almost everyone is now looking for the next opportunity.

Annular eclipses are different from the total eclipses only in geometry. The angular size of the Moon is just not sufficient to block the Sun, which shines like a band outside the dark orb of the Moon. This is a simple consequence of their relative distances. For example on $15^{\text {th }}$ January Moon was farthest at 4,06,125 km (farther than the average distance of 3,84,000 km) and hence looked smaller than normal. Such differences in size are barely noticeable with naked eye. On the other hand, the full Moon of $30^{\text {th }}$ was the closest, perigee occurring within 3 hours of the full Moon. Thus, the full Moon appeared larger than normal. A direct consequence of this is on the tides, which will be higher than normal. The Sun also displays a variation in its size annually. On $3^{\text {rd }}$ January it was nearest and will be farthest on $4^{\text {th }}$ July. On the $15^{\text {th }}$ January it was at a distance of $148,006,650 \mathrm{~km}$, which puts it nearer than normal. Again, this difference is not noticeable to the naked eye. This results in a slightly larger disc of the Sun. The Moon is not able to cover the Sun completely; the eclipse is thus an annular eclipse.

The Sun looking like a ring in the sky must have fascinated laypersons in the past. We find documentations in various forms. For example, a stone inscription in Ottur near Soraba in Karnataka records a "Valaya grahana" which corroborates with annular eclipse of February 3, 939AD. Similarly, a correspondence in the journal Observatory dated April 1901, discusses the record of annular solar eclipse on October 7, $218 \mathrm{AD}$ in book by the Greek historian Dion Cassius. Analyses of ancient solar eclipse records in literature probes into the tendency of secular changes in the rotation of the earth. (Stephenson, F.R., 1997, "Historical eclipses and Earth's rotation, Cambridge University Press; Zhang P, 1994, Publ Purple Mt Observatory 13, 23Z). Generally, annular eclipses are not of great interest to solar physicists since the corona and chromospheres are not seen. Even just eight percent of the photosphere visible at totality is sufficient to hide these two regions of the Sun. Thus, there is no scope to do those experiments slated for the total eclipse.

On the other hand, every eclipse provides interesting opportunity for the study of our home, the Earth. One of the earliest records dated April 6, 1894, of the observations made from Madras in India; the observation team headed by Michie Smith included R.L. Jones and K.V. Sivaramaiah. The annularity was visible from Cuddapah. The report says ".....none of them contained any feature of special interest". The meteorological observations were measurement of temperature 
(a dry and a wet thermometer in the shade), humidity and pressure (MNRAS, 1894, 55, 76-77). The records show a dip of 5 degrees in temperature. Donitch of Russia, was to observe the solar annular eclipse, which was visible from Malacca, French Indochina, Cambodia and Annam on 1617 March 1904. De la Baume Pluvinel in Egypt had observed the previous annular eclipse on 11 November 1901. Donitch made use of the experience of his predecessor. He utilized a photographic telescope, a Lippmann coelostat, and a Zeiss microscope of a variable enlargement (for the photographs examination) and a thermometer. He travelled to Pnom-Penh in Cambodia, on the central line of eclipse. He observed the reversal of the black lines in the spectroscope attached to the spectrograph. The temperature variation was considerable during the two and a half hours of the eclipse. The maximal fall of temperature being 3 degrees (1905, Izv Russian Astron Obs, 10, 276)

In the last few decades annular eclipses have been utilized for parameters pertaining to the Earth. During the annular eclipse of September 23, 1989, several kinds of observations, such as doppler measurements were carried out in a three-station network. Also rapid-run ionosonde, were simultaneously carried out at Wuhan Ionospheric Observatory to observe the ionospheric effects of the eclipse. Both the horizontal and the vertical propagation of the travelling ionospheric disturbances (TIDs) after the solar eclipse was investigated in detail. It was found that the TIDs in different ionospheric heights propagated to, instead of from, the central band of the eclipse with downward phase velocities. This may imply that the sources of the TIDs are located in the lower atmosphere far from the eclipse band (1990, Li, Wan, Wu, Ning and Li, Acta Geophys. Sin, 33, 384).

In yet another annular eclipse of April 29, 1976, the time variation of ionospheric absorption at frequencies of $1.8,2.2$, and $2.6 \mathrm{MHz}$ over Ashkhabad, $\left(38^{\circ} \mathrm{N}, 58^{\circ} \mathrm{E}\right)$, was examined. A slight decrease in absorption with increasing frequency was found along with a moderate increase in E-layer reflection height at all three frequencies. It was shown that at the maximum phase of the eclipse, ionospheric absorption attained a minimum value of $4 \mathrm{~dB}$, which corresponds to nighttime conditions (1978 Ge and Ae, Shirmammedov, Bolatev, Geomagnetizm I Aeronomiia, 18, 737). A detailed study of the E-layer assumed that these spectral variations in the parameters of the $\mathrm{E}_{\mathrm{s}}$-layer are caused by gravitational waves generated by the solar eclipse (Kardashev, Y, Gorbunova, T A, 1990, Ge and Ae, 30, 875).

The ionospheric effects of the annular eclipse of September 23, 1989 observed by HF skywave backscatter radar, show that the irregularity and the small-scale ripples existed on the annular eclipse path during the solar eclipse (Jiao, P, 1990, Acta Geophys Sin 33, 391).

By using the data on solar radiation and surface meteorological elements observed during the annular eclipses, it was found that apart from the variation of solar radiation and meteorological elements, the variation of the ground ozone concentration was also noticeable during the annular eclipse.

Two or more (in rare cases as many as five) solar eclipses are observed on Earth every year. However, solar eclipses for a particular point on Earth occur more rarely. The average interval 
between two such events is about half a century. The relation between the position and the size of the Moon and the occurrence and characteristics of solar eclipses is considered. It is found that only total solar eclipses would be observed if the distance between Earth and Moon were reduced by approximately 10 percent. Only annular eclipses would occur if the distance of the Moon were about 6 percent greater. It is pointed out that a sharp distinction between total and annular eclipses can only be made from a mathematical point of view. However, the limb of the Moon is not an ideal circle, but a line characterized by mountains and valleys. These relations give rise to the observation of Baily's beads.

Studies related to the Sun are not ruled out, for example, one can measure the limb darkening and the effect of faculae (these are bright regions on the photosphere). During the annular eclipse of 19 April 1958, measurements were made with a photoelectric device. Results were directly compared with the total flux from uneclipsed Sun; there was no effect of faculae on limb darkening (Saito, K. and Hata, S., 1960, PASJ, 12, 143).

In the 1920s many were speculating the predictions of Einstein's theory. One of the papers discusses the point in the context of annular eclipses; it reads - "As I said ........... the refractivity $\mu$, necessary at every point of a gravitational field to produce the Einstein deflection, is the ratio of the energy of a constant-mass particle fallen there from infinity to the energy of the same particle moving with the speed of light; but it is not permissible to say that the solar gravitational field acts like a lens, for it has no focal length. If the Sun were backed by a nebula or any luminous area, the light grazing the rim all round would be brought to a focus at a place seventeen times the distance of Neptune, while light from any larger circle would focus still further off in proportion to the area of the circle. So from a uniformly luminous area there would result a focal line of constant brightness. The Moon is, unfortunately, impotent to make an annular eclipse interesting" (Oliver Lodge, 1919, Nature, 104, 354).

Annular eclipse provides a very good opportunity to measure the lunar limb profile and the diameter of the Sun precisely.

During the past 20 years, timings of eclipse 2nd and 3rd contacts, and other Baily's bead phenomena, have been made near the edges of the paths of totality and annularity of several solar eclipses using visual, photographic, and video observations of the direct or projected solar image formed with telescopes. Historical observations of sufficient accuracy made near total eclipse limits have been found in the literature going back to 1715; most of these are direct visual observations. Analyses of these observations have revealed probable small variations of the solar diameter. Results from the past century can be compared with global weather observations to help resolve the question of whether global temperature changes are caused more by natural solar variations or by anthropomorphic causes such as increased burning of fossil fuel. Most of the recent effort has been directed towards recording the Baily's bead phenomena more accurately, but it is clear that the new techniques must be compared with those used for historical eclipses, so visual observations are still needed for this calibration (1995, Dunham, D., Fiala, A. and Sofia, S., AAS, 187.020). 
Radio measurements are made during the eclipse to get size of the "radio sun" as was done by Ji, D.S., Liang, S.G. (1990, Prog. Astron., 8, 173).

The first hint of the fact that solar eclipses mark the enhanced storms called El Nino or La Nina, came from the article by Robert Allan on analysis of frequencies of these events. One mystery was the cause of a cycle with period 15 to 20 years. But the Saros Series of solar eclipses has a period of 18 years.

The data from Galapagos Islands for the whole 20th century came very handy. A search through charts of solar eclipses for those with good locations for bringing high tides at the Tropics, gave a good picture: those at the eastern coast of the Pacific Ocean gave El Ninos, and those at the west gave La Ninas. A closer look at a few events that caused great storms are described in J.M. Nash's book, "El Nino" (2002). The most striking case is that of the 1998 February 22 solar eclipse, which corresponds to the so-called El Nino of 1997-98. Thus, now it is being speculated that the annual El Nino effect is due to the Sun's travel between the Tropic of Cancer and Tropic of Capricorn; But the enhanced El Nino/La Nina is due to the coming together of Sun and Moon in the solar eclipses, which seem to come irregularly (Chiu B C, 2005, AAAS, 37.5002).

Eclipse expeditions are not new to India. During the colonial days, several European teams visited India for eclipses. The discovery of helium in the Sun during the 1868 eclipse was made when the Moon's shadow track passed through the fields of South India.

This eclipse of 15 January 2010 has raised considerable interest among scientists of various disciplines. Various experiments on light, (visible and ultra violet); temperature, and even wind pattern variation have been attempted. Also of interest are the behaviour of plants, animals and insects too. Let us look forward to some interesting results which will help in demystifying the beautiful celestial event.

Jawaharlal Nehru Planetarium

B. S. SHYLAJA

High Grounds, Bangalore

Email: shylaja.jnp@gmail.com 